\title{
Time History Interfacial Conditions in Multiscale Computations of Lattice Oscillations
}

\author{
Michael Dreher and Shaoqiang Tang
}

February 28, 2007

\begin{abstract}
We make a systematic study on the time history interfacial conditions in multiscale computations for crystalline solids in one space dimension. The exact interfacial condition is derived rigorously. For a class of approximate time history kernel functions, the error is estimated. In particular, a cut-off of the exact kernel function is a special case. Moreover, an effective numerical algorithm is proposed for the convolution.
\end{abstract}

\section{Introduction}

A uniform periodic lattice structure is usually assumed for a bulk material in the studies of solid state physics or elasticity [3]. This allows the application of various mathematical tools. However, when there are defects, such as dislocations or phase interfaces, the uniform infinite structure does not correctly represent the reality [9]. Under such circumstances, a multiscale methodology may be adopted to reach a compromise between the numerical accuracy and efficiency [7]. In a concurrent multiscale algorithm, one treats a selected small subdomain enclosing the defects by the detailed atomistic dynamics, whereas resolves the vast surrounding region by a coarse grid or continuum description. The quality of such an algorithm heavily relies on the interfacial condition that is imposed across the different scales. A class of such interfacial conditions, called a time history treatment, were proposed first by Adelman and Doll [2], and adopted for multiscale computations by Cai et al. in [4]. A displacement decomposition has been further incorporated in a Bridging Scale Method [12, 10] and a Pseudo-spectral Multiscale Method [11]. Other numerical methods with similar strategy have also been developed [6].

As we shall show in the following discussion, an exact time history treatment requires computing a convolution for the whole time history, which is virtually forbidden in most implementations. Therefore, one usually makes a certain truncation (cut-off) in the time history to save the storage and computing load. The influence of such truncation is largely unclear. In particular, reflections after a long run have been observed by various researchers, when such truncations are performed.

In this paper, we shall rigorously derive the interfacial conditions for a semi-infinite lattice in one space dimension, and estimate the error caused due to the approximation of the time history 
kernels. For a simple truncation, we discover the sensitivity on the choice of the cut-off time. Moreover, a more accurate approximate time history kernel function is suggested, making use of the asymptotics for the Bessel function.

The rest of the paper is planned as follows. First, we derive in Section 2 the solution representation for an infinite ODE system describing the harmonic lattice. Then we analyze in Section 3 the error for numerical schemes with an approximate kernel function. In particular, the truncated convolution corresponds to a special choice for the approximate kernel. Our main results are Theorem 3.4 with detailed error estimates, and a fast algorithm presented in Section 3.3 for evaluating convolution integrals over long intervals.

\section{Solution Representations for Infinite and Semi-infinite Lat- tices}

In a harmonic lattice, we write $u_{n}$ for the displacement away from the equilibrium at the $n$-th atom. After rescaling, the Newton law reads

$$
u_{n}^{\prime \prime}=u_{n-1}-2 u_{n}+u_{n+1} .
$$

For the infinite and semi-infinite lattices, we define infinite or semi-infinite displacement vectors

$$
u=\left(u_{n}\right)_{n \in \mathbb{Z}}, \quad u_{>b}=\left(u_{n}\right)_{n>b}=\left(u_{b+1}, u_{b+2}, \ldots\right)^{\top}, \quad u_{\leq b}=\left(u_{n}\right)_{n \leq b}=\left(\ldots, u_{b-1}, u_{b}\right)^{\top} .
$$

Here $b \in \mathbb{Z}$ marks the boundary of the semi-infinite lattices. We further define three infinite or semi-infinite matrices as follows.

$$
\begin{aligned}
& A=\left(a_{i j}\right)_{i, j \in \mathbb{Z}}, \quad A^{+}=\left(a_{i j}\right)_{i, j \in \mathbb{N}_{+}}, \quad A^{-}=\left(a_{i j}\right)_{-i,-j \in \mathbb{N}_{+}}, \\
& a_{i j}= \begin{cases}2 \quad: i=j \\
-1 & :|i-j|=1 \\
0 \quad & : \text { else. }\end{cases}
\end{aligned}
$$

The infinite lattice is governed by

$$
\left\{\begin{array}{l}
\partial_{t}^{2} u(t)=-A u(t), \\
u(0)=u^{(0)}, \quad u^{\prime}(0)=u^{(1)} .
\end{array}\right.
$$

The right semi-infinite lattice is governed by

$$
\left\{\begin{array}{l}
\partial_{t}^{2} u_{>b}=-A^{+} u_{>b}+G, \\
u_{>b}(0)=u_{>b}^{(0)}, \quad u_{>b}^{\prime}(0)=u_{>b}^{(1)},
\end{array}\right.
$$

with a vector $G=(g, 0,0, \ldots)^{\top}$ to be specified. 
The left semi-infinite lattice is governed by

$$
\left\{\begin{array}{l}
\partial_{t}^{2} u_{\leq b}=-A^{-} u_{\leq b}+G, \\
u_{\leq b}(0)=\left(u_{n}^{(0)}\right)_{n \leq b}, \quad u_{\leq b}^{\prime}(0)=\left(u_{n}^{(1)}\right)_{n \leq b},
\end{array}\right.
$$

where $G=(\ldots, 0,0, g)^{\top}$ is also to be specified.

Applying the Fourier transform

$$
u=\left(u_{n}\right)_{n \in \mathbb{Z}} \mapsto \hat{u}=\hat{u}(\varphi)=\sum_{n \in \mathbb{Z}} u_{n} \exp (-\mathrm{i} \varphi n), \quad \varphi \in[-\pi, \pi],
$$

to the infinite lattice problem (2.2), we get a second order ODE with parameter $\varphi$,

$$
\partial_{t}^{2} \hat{u}(t, \varphi)=-(2-2 \cos \varphi) \hat{u}(t, \varphi) .
$$

Solving this ODE and performing the inverse Fourier transform, we obtain the following representation formula.

$$
u_{n}(t)=\sum_{m \in \mathbb{Z}}\left(u_{m}(0) J_{2(n-m)}(2 t)+u_{m}^{\prime}(0) \sum_{l=|n-m|}^{\infty} J_{2 l+1}(2 t)\right), \quad n \in \mathbb{Z} .
$$

Here, we have exploited (9.1.42) from [1],

$$
\cos (z \sin \theta)=J_{0}(z)+2 \sum_{l=1}^{\infty} J_{2 l}(z) \cos (2 l \theta)
$$

with $J$ the Bessel functions, which satisfy $J_{-n}(z)=(-1)^{n} J_{n}(z)$ and $J_{\nu-1}(z)-J_{\nu+1}(z)=2 J_{\nu}^{\prime}(z)$. We summarize the results as follows.

Proposition 2.1. For any pair of vectors $u^{(0)}=\left(u_{n}^{(0)}\right)_{n \in \mathbb{Z}}, u^{(1)}=\left(u_{n}^{(1)}\right)_{n \in \mathbb{Z}} \in l^{2}(\mathbb{Z})$, (2.5) gives the unique solution $u=\left(u_{n}(t)\right)_{n \in \mathbb{Z}} \in C^{2}\left(\mathbb{R}, l^{2}(\mathbb{Z})\right)$ to the system $(2.2)$.

Next, we consider the dynamics for the semi-infinite lattice governed by (2.3).

Lemma 2.2 (Fundamental solution to the right half-space problem). There is a $\mathbb{N}_{+} \times \mathbb{N}_{+}$ matrix function $\mathcal{U}^{+}=\mathcal{U}^{+}(t)$ with the property that $u_{>b}(t):=\mathcal{U}^{+}(t) \cdot u_{>b}^{(1)}$ solves problem $(2.3)$ with $G=0, u_{>b}^{(0)}=0$, and $u_{>b}^{(1)} \in l^{2}$. The entries of $\mathcal{U}^{+}$are

$$
\mathcal{U}_{n m}^{+}(t)=\sum_{l=|n-m|}^{n+m-1} J_{2 l+1}(2 t), \quad n, m \geq 1
$$

Proof. Fix initial data $v_{n}^{(0)}=0$ for $n \in \mathbb{Z}$, and

$$
v_{n}^{(1)}= \begin{cases}u_{n}^{(1)} & : n>b \\ 0 & : n=b \\ -u_{2 b-n}^{(1)} & : n<b\end{cases}
$$




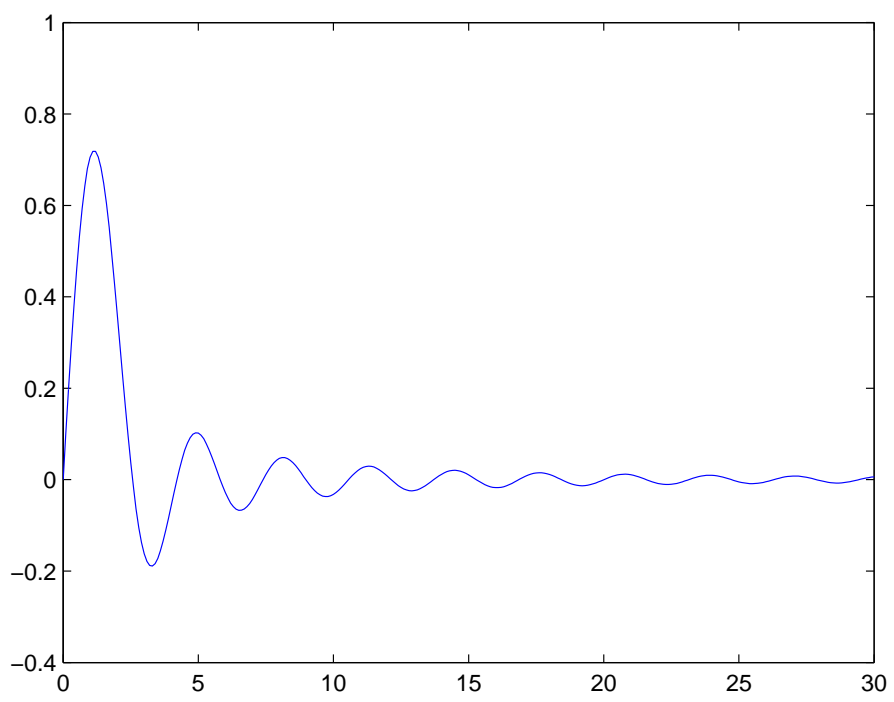

Figure 1: The function $\mathcal{U}_{11}^{+}$

and define a function $v=v(t)=\left(v_{n}(t)\right)_{n \in \mathbb{Z}}$ via

$$
v_{n}(t)=\sum_{m \in \mathbb{Z}} v_{m}^{(1)}\left(\sum_{l=|n-m|}^{\infty} J_{2 l+1}(2 t)\right) .
$$

From (2.5) we see that this vector $v$ is the unique solution to the full-space problem (2.2) with initial values $u^{(0)}=0$ and $u^{(1)}=v^{(1)}$. By a reflection argument and the uniqueness, we see that $v$ is odd at $n=b$ for all times: $v_{n}(t)=-v_{2 b-n}(t), n \in \mathbb{Z}$. Therefore, we know that $u_{n}(t)=v_{n}(t)$, for $n \geq b$. The formula (2.6) then follows from a comparison of (2.7) with the relation defining $u_{>b}(t)$,

$$
u_{b+n}(t)=\sum_{m=1}^{\infty} \mathcal{U}_{n m}^{+}(t) u_{b+m}^{(1)}, \quad n \geq 1
$$

Proposition 2.3 (Duhamel's principle). The solution $u_{>b}$ to $(2.3)$ is

$$
\begin{aligned}
& u_{>b}(t)=\left(\partial_{t} \mathcal{U}^{+}(t)\right) \cdot u_{>b}^{(0)}+\mathcal{U}^{+}(t) \cdot u_{>b}^{(1)}+\int_{t^{\prime}=0}^{t} \mathcal{U}^{+}\left(t-t^{\prime}\right) \cdot G\left(t^{\prime}\right) \mathrm{d} t^{\prime}, \\
& u_{b+n}(t)=\sum_{m=1}^{\infty}\left(\partial_{t} \mathcal{U}_{n m}^{+}(t) u_{b+m}^{(0)}+\mathcal{U}_{n m}^{+}(t) u_{b+m}^{(1)}+\int_{t^{\prime}=0}^{t} \mathcal{U}_{n m}^{+}\left(t-t^{\prime}\right) G_{b+m}\left(t^{\prime}\right) \mathrm{d} t^{\prime}\right), \quad n \geq 1 .
\end{aligned}
$$

By well-known formulas on recurrence relations and Laplace transforms of Bessel functions [1], the following properties of the entries $\mathcal{U}_{n m}^{+}$are easily shown. 
Lemma 2.4. The function $\mathcal{U}_{11}^{+}$is given by $\mathcal{U}_{11}^{+}(t)=\frac{2}{t} J_{2}(2 t)$.

For $n, m \geq 1$, we have $\mathcal{U}_{n m}^{+}(t)=\mathcal{U}_{m n}^{+}(t)$ and $\partial_{t} \mathcal{U}_{n m}^{+}(t)=J_{2|n-m|}(2 t)-J_{2(n+m)}(2 t)$.

For $n \geq 1$, the Laplace transform $\hat{\mathcal{U}}_{n 1}^{+}$of $\mathcal{U}_{n 1}^{+}$is

$$
\hat{\mathcal{U}}_{n 1}^{+}(s)=\left(\frac{s}{2}+\sqrt{1+\frac{s^{2}}{4}}\right)^{-2 n}=\left(\frac{s}{2}-\sqrt{1+\frac{s^{2}}{4}}\right)^{2 n}, \quad s \geq 0 .
$$

Remark 2.5. A direct consequence are the convolution identities $\mathcal{U}_{n 1}^{+} * \mathcal{U}_{m 1}^{+}=\mathcal{U}_{n+m, 1}^{+}$.

In the same way, we may treat $u_{\leq b}$ and obtain a representation as follows.

Corollary 2.6 (Duhamel's Principle). The solution $u_{\leq b}$ to $(2.4)$ is

$$
u_{b+1+n}(t)=\sum_{m=-1}^{-\infty}\left(\partial_{t} \mathcal{U}_{n m}^{-}(t) u_{b+1+m}^{(0)}+\mathcal{U}_{n m}^{-}(t) u_{b+1+m}^{(1)}+\int_{t^{\prime}=0}^{t} \mathcal{U}_{n m}^{-}\left(t-t^{\prime}\right) G_{b+1+m}\left(t^{\prime}\right) \mathrm{d} t^{\prime}\right)
$$

where $n \leq-1$. Here the entries $\mathcal{U}_{n m}^{-}$of $\mathcal{U}^{-}$are given by $\mathcal{U}_{n m}^{-}(t)=\mathcal{U}_{-n,-m}^{+}(t)$, for $n, m \leq-1$.

\section{Error Estimates for Approximate Half-Space Problems}

\subsection{A General Scheme}

In a multiscale computation, one reduces the computing load by taking a coarse grid representation away from a selected small subdomain. We idealize the subdomain as $u_{\leq b}$ with the $b$-th atom being the interface. In such a selection, fine fluctuations are assumed to be generated within the subdomain only. An interfacial condition is prescribed at the $b$-th atom to close the atomistic dynamics in such a way that all waves from $u_{\leq b}$ pass to $u_{>b}$ without reflection.

Due to the linearity, superposition applies for the harmonic lattice. The non-reflecting interfacial condition may be designed together with a displacement decomposition $[10,11]$. In this setting, we consider the full space problem (2.2), which is initially at equilibrium away from the atomistic subdomain. This means $u_{n}^{(0)}=u_{n}^{(1)}=0$ for $n \geq b \in \mathbb{Z}$.

Then the semi-infinite vector $u_{>b}$ solves the right half-space problem (2.3) with vanishing initial data and $G=(g, 0,0, \ldots)^{\top}$, where $g(t)=u_{b}(t)$. From the Duhamel principle for the right half-space problem, we then have

$$
u_{b+1}(t)=\left(\mathcal{U}_{11}^{+} * u_{b}\right)(t)=\int_{t^{\prime}=0}^{t} \frac{2}{t^{\prime}} J_{2}\left(2 t^{\prime}\right) u_{b}\left(t-t^{\prime}\right) \mathrm{d} t^{\prime}
$$

Let the vector $u_{\leq b}$ be the solution to the problem with exact interface conditions

$$
\left\{\begin{array}{l}
\partial_{t}^{2} u_{\leq b}=-A^{-} u_{\leq b}+G_{(u)}, \\
u_{\leq b}(0)=\left(u_{n}^{(0)}\right)_{n \leq b}, \quad u_{\leq b}^{\prime}(0)=\left(u_{n}^{(1)}\right)_{n \leq b}, \\
G_{(u)}=\left(\ldots, 0, \ldots, 0, g_{(u)}\right)^{\top}, \quad g_{(u)}(t)=u_{b+1}(t)=\left(\mathcal{U}_{11}^{+} * u_{b}\right)(t) .
\end{array}\right.
$$


Let $\tilde{\mathcal{U}}_{11}^{+}$be an approximation of the convolution kernel $\mathcal{U}_{11}^{+}$. Define a convolution operator $R$ as $(R * z)(t):=\left(\mathcal{U}_{11}^{+} * z\right)(t)-\left(\tilde{\mathcal{U}}_{11}^{+} * z\right)(t)$.

Let $v_{\leq b}$ be the solution to the approximate problem with the same initial values,

$$
\left\{\begin{array}{l}
\partial_{t}^{2} v_{\leq b}=-A^{-} v_{\leq b}+G_{(v)}, \\
v_{\leq b}(0)=\left(u_{n}^{(0)}\right)_{n \leq b}, \quad v_{\leq b}^{\prime}(0)=\left(u_{n}^{(1)}\right)_{n \leq b} \\
G_{(v)}=\left(\ldots, 0, \ldots, 0, g_{(v)}\right)^{\top}, \quad g_{(v)}(t)=\left(\tilde{\mathcal{U}}_{11}^{+} * v_{b}\right)(t)
\end{array}\right.
$$

Then we define the error vector $w_{\leq b}:=u_{\leq b}-v_{\leq b}$, which is a solution to

$$
\left\{\begin{array}{l}
\partial_{t}^{2} w_{\leq b}=-A^{-} w_{\leq b}+G_{(w)} \\
w_{\leq b}(0)=w_{\leq b}^{\prime}(0)=0 \\
G_{(w)}=\left(\ldots, 0, \ldots, 0, g_{(w)}\right)^{\top}
\end{array}\right.
$$

with

$$
g_{(w)}(t)=g_{(u)}(t)-g_{(v)}(t)=\left(\mathcal{U}_{11}^{+} * w_{b}\right)(t)+\left(R * v_{b}\right)(t)=\left(\tilde{\mathcal{U}}_{11}^{+} * w_{b}\right)(t)+\left(R * u_{b}\right)(t) .
$$

Our goal is to estimate the approximation error $w_{\leq b}$ in terms of $u_{\leq b}$. To this end, we apply Corollary 2.6 to the system (3.3), and find, by a result for the left semi-infinite lattice similar to Lemma 2.2, the representations

$$
\begin{aligned}
& w_{b+1+n}(t)=\left(\mathcal{U}_{n,-1}^{-} * g_{(w)}\right)(t), \quad n \leq-1, \\
& w_{b}(t)=\left(\mathcal{U}_{11}^{+} * g_{(w)}\right)(t)=\left(\mathcal{U}_{11}^{+} *\left(\mathcal{U}_{11}^{+}-R\right) * w_{b}\right)(t)+\left(\mathcal{U}_{11}^{+} * R * u_{b}\right)(t),
\end{aligned}
$$

from which we deduce, using (2.8), that

$$
\hat{w}_{b}(s)=\frac{1}{s \sqrt{4+s^{2}}+\hat{R}(s)} \hat{R}(s) \hat{u}_{b}(s)=: \hat{K}(s) \hat{R}(s) \hat{u}_{b}(s) .
$$

Note the zeros of $s \sqrt{4+s^{2}}$ at $s=0$ and $s= \pm 2$ i. For brevity, we introduce the notation

$$
f=f(s):=s \sqrt{4+s^{2}}, \quad \sigma_{ \pm}:= \pm 2 \mathrm{i} .
$$

Next, we are going to answer the question whether this newly defined function $\hat{K}$ is the Laplace transform of a function $K$. To this end, we make the entirely reasonable assumption that $R=R(t)$ is at most exponentially growing for $t \rightarrow \infty$, with growth rate $s_{0} \in \mathbb{R}$. Then the Laplace transform $\hat{R}=\hat{R}(s)$ exists for all $s \in \mathbb{C}$ with $\Re s \geq s_{0}$, it is an analytic function of $s$ for $\Re s>s_{0}$, and we have a bound $|\hat{R}(s)| \leq C_{0}$ for $\Re s \geq s_{0}$. Then there is a real number $s_{1} \geq s_{0}$ such that $\left|s \sqrt{4+s^{2}}\right| \geq 2 C_{0}$ for $\Re s \geq s_{1}$, which has the following consequences:

- $\hat{K}=\hat{K}(s)$ is an analytic function of $s$ for $\Re s>s_{1}$,

- for any $\delta>0, \hat{K}(s)$ converges uniformly to zero for $s \rightarrow \infty, \Re s \geq s_{1}+\delta$,

- for any $\delta>0$, the line integral $\int_{s=s_{1}+\delta-\mathrm{i} \infty}^{s=s_{1}+\delta+\mathrm{i}}|\hat{K}(s)| \mathrm{d} s$ is bounded. 
Then (see [5, Vol.I]), the function $\hat{K}$ is the Laplace transform of a function $K=K(t)$,

$$
K(t)=\frac{1}{2 \pi \mathrm{i}} \int_{s=s_{1}+\delta-\mathrm{i} \infty}^{s_{1}+\delta+\mathrm{i} \infty} e^{t s} \hat{K}(s) \mathrm{d} s, \quad t \geq 0, \quad \delta>0 .
$$

This allows us to write

$$
w_{b}(t)=u_{b}(t)-v_{b}(t)=\left(K * R * u_{b}\right)(t), \quad t \geq 0,
$$

from which estimates $\left\|w_{b}\right\|_{L^{p}\left(\mathbb{R}_{+}\right)} \leq\|K * R\|_{L^{1}\left(\mathbb{R}_{+}\right)}\left\|u_{b}\right\|_{L^{p}\left(\mathbb{R}_{+}\right)}$can be deduced for any $1 \leq p \leq \infty$ provided that $K * R \in L^{1}\left(\mathbb{R}_{+}\right)$and $u_{b} \in L^{p}\left(\mathbb{R}_{+}\right)$.

Lemma 3.1. The function $w_{b}$ solves the differential equation

$$
\partial_{t}^{2} w_{b}=\left(2 \mathcal{U}_{11}^{+}-R\right) * w_{b}-2 w_{b}+R * u_{b}
$$

Proof. From (3.4), Remark 2.5, and a result for the left semi-infinite lattice similar to Lemma 2.2, we know that

$$
w_{b-1}(t)=\left(\mathcal{U}_{21}^{-} * g_{(w)}\right)(t)=\left(\mathcal{U}_{11}^{+} * \mathcal{U}_{11}^{+} * g_{(w)}\right)(t)=\left(\mathcal{U}_{11}^{+} * w_{b}\right)(t) .
$$

On the other hand, $w_{b}$ solves a differential equation, via (3.3):

$$
\partial_{t}^{2} w_{b}=w_{b-1}-2 w_{b}+g_{(w)}=\left(2 \mathcal{U}_{11}^{+}-R\right) * w_{b}-2 w_{b}+R * u_{b} .
$$

Lemma 3.2. Let $p_{\alpha}$ denote the function with $p_{\alpha}(t)=t^{\alpha}$, for $\alpha \in \mathbb{R}$. Then the functions $K$ and $R * K$ can be expressed as

$$
\begin{aligned}
K(t) & =t+\left(p_{1} *\left(2 \mathcal{U}_{11}^{+}-R\right) * K\right)(t)-2\left(p_{1} * K\right)(t), \\
(R * K)(t) & =\left(p_{1} * R\right)(t)+\left(p_{1} *\left(2 \mathcal{U}_{11}^{+}-R\right) * R * K\right)(t)-2\left(p_{1} * R * K\right)(t) .
\end{aligned}
$$

Proof. The function $p_{1}$ has the Laplace transform $\hat{p}_{1}(s)=s^{-2}$. Then we can write

$$
\begin{aligned}
K(t) & =t+\int_{s=s_{1}+\delta-\mathrm{i} \infty}^{s_{1}+\delta+\mathrm{i} \infty} e^{t s} \frac{1}{s^{2}}\left(s^{2} \hat{K}(s)-1\right) \mathrm{d} s \\
& =t+\int_{s=s_{1}+\delta-\mathrm{i} \infty}^{s_{1}+\delta+\mathrm{i} \infty} e^{t s} \frac{1}{s^{2}}\left(2 \hat{\mathcal{U}}_{11}^{+}(s)-\hat{R}(s)-2\right) \hat{K}(s) \mathrm{d} s \\
& =t+\left(p_{1} *\left(2 \mathcal{U}_{11}^{+}-R\right) * K\right)(t)-2\left(p_{1} * K\right)(t) .
\end{aligned}
$$

Convolving this first identity with $R$ gives the second assertion.

Corollary 3.3. The functions $K$ and $R * K$ solve the initial-value problems

$$
\begin{aligned}
& K^{\prime \prime}(t)=\left(\left(2 \mathcal{U}_{11}^{+}-R\right) * K\right)(t)-2 K(t), \quad K(0)=0, \quad K^{\prime}(0)=1, \\
& (R * K)^{\prime \prime}(t)=R(t)+\left(\left(2 \mathcal{U}_{11}^{+}-R\right) *(R * K)\right)(t)-2(R * K)(t), \quad(R * K)(0)=(R * K)^{\prime}(0)=0 .
\end{aligned}
$$




\subsection{An Example for the Approximate Kernel}

The goal of this section is to propose a class of approximate kernels $\tilde{\mathcal{U}}_{11}^{+}$and to estimate the factor $\|K * R\|_{L^{p}}$ which enters in the error estimate as good as possible. To this end, we start with recalling that the Bessel functions satisfy the asymptotic expansion

$$
J_{\nu}(t)=\sqrt{\frac{2}{\pi t}}(P(\nu, t) \cos \chi(\nu, t)-Q(\nu, t) \sin \chi(\nu, t)), \quad t \rightarrow+\infty
$$

where $\chi(\nu, t)=t-(\nu / 2+1 / 4) \pi$ and

$$
P(\nu, t) \sim \sum_{l=0}^{\infty} a_{\nu l} t^{-2 l}, \quad Q(\nu, t) \sim \frac{1}{2 t} \sum_{l=0}^{\infty} b_{\nu l} t^{-2 l},
$$

and the constants $a_{\nu l}, b_{\nu l}$ are

$$
a_{\nu l}=\frac{(-1)^{l}}{2^{2 l}} \frac{\Gamma(\nu+2 l+1 / 2)}{(2 l) ! \Gamma(\nu-2 l+1 / 2)}, \quad b_{\nu l}=\frac{(-1)^{l}}{2^{2 l}} \frac{\Gamma(\nu+2 l+3 / 2)}{(2 l+1) ! \Gamma(\nu-2 l-1 / 2)} .
$$

The error arising from truncating the asymptotic series after $l$ is smaller than the first skipped term provided that $t \in \mathbb{R}, l>\nu / 2-1 / 4$ for $P$, and $l>\nu / 2-3 / 4$ for $Q$.

This suggests the following choice of a kernel $\tilde{\mathcal{U}}_{11}^{+}$approximating $\mathcal{U}_{11}^{+}(t)=2 J_{2}(2 t) / t$ : choose a cut-off time $T>0$ and an integer $k^{\prime} \geq-1$, and set

$$
\tilde{\mathcal{U}}_{11}^{+}(t):= \begin{cases}\mathcal{U}_{11}^{+}(t) & : 0 \leq t<T \\ \frac{2}{\sqrt{\pi}} t^{-3 / 2} \sum_{l=0}^{k^{\prime}}\left(\frac{a_{2 l}}{(2 t)^{2 l}} \cos \left(2 t-\frac{5}{4} \pi\right)-\frac{1}{4 t} \frac{b_{2 l}}{(2 t)^{2 l}} \sin \left(2 t-\frac{5}{4} \pi\right)\right) & : T \leq t<\infty\end{cases}
$$

If $k^{\prime}=-1$, then $\tilde{\mathcal{U}}_{11}^{+}(t)=0$ for $T \leq t<\infty$. The remainder $R=\mathcal{U}_{11}^{+}-\tilde{\mathcal{U}}_{11}^{+}$vanishes for $0 \leq t<T$. Put $k=2 k^{\prime}+3$.

As an example, we choose $k=1$ and plot the functions $K$ for various cut-off times $T$ in Figure 2 . The plots are obtained by transforming the second-order differential equation from Corollary 3.3 into a first-order system, which is then solved numerically using the implicit trapezoidal method with step-size 0.05; and the Simpson method chosen for the quadrature of the convolutions.

In the sequel, we will show that the function $K$ is a sum of two terms: the first is either slowly exponentially decaying or increasing (depending on the choice of $k$ and $T$ ), and the second is oscillating and decaying at least like $t^{-1}$ for $t \rightarrow+\infty$. We will present a precise description of the first term, from which we will learn how to choose good values for $k$ and $T$. Our pointwise estimates of $K * R$ will then lead naturally to the following main result:

Theorem 3.4. Let $u_{\leq b}$ be the solution to the exact system (3.1), with the convolution kernel $\mathcal{U}_{11}^{+}$ given as in Lemma 2.4. Let $v_{\leq b}$ be the solution to approximate system (3.2), with a convolution kernel $\tilde{\mathcal{U}}_{11}^{+}$chosen in (3.5), and let $w_{\leq b}=u_{\leq b}-v_{\leq b}$ denote the error.

For the numbers $k=2 k^{\prime}+3$ and $T$ from (3.5), we assume $k \equiv 1 \bmod 4, T \gg 1$, and that $2 T-\pi / 4$ is situated near a maximum of the sine function. Then we have the following error estimates, for any interval $\left(0, T_{0}\right)$ :

$$
\begin{aligned}
& \left\|w_{b}\right\|_{L^{\infty}\left(0, T_{0}\right)} \leq C T^{-k / 2}\left\|u_{b}\right\|_{L^{2}\left(0, T_{0}\right)}, \\
& \left\|w_{b}\right\|_{L^{\infty}\left(0, T_{0}\right)} \leq C T^{-k}\left\|u_{b}\right\|_{L^{1}\left(0, T_{0}\right)}, \\
& \left\|w_{b}\right\|_{L^{p}\left(0, T_{0}\right)} \leq C \ln \left(1+T_{0} T^{-k}\right)\left\|u_{b}\right\|_{L^{p}\left(0, T_{0}\right)}, \quad 1 \leq p \leq \infty .
\end{aligned}
$$



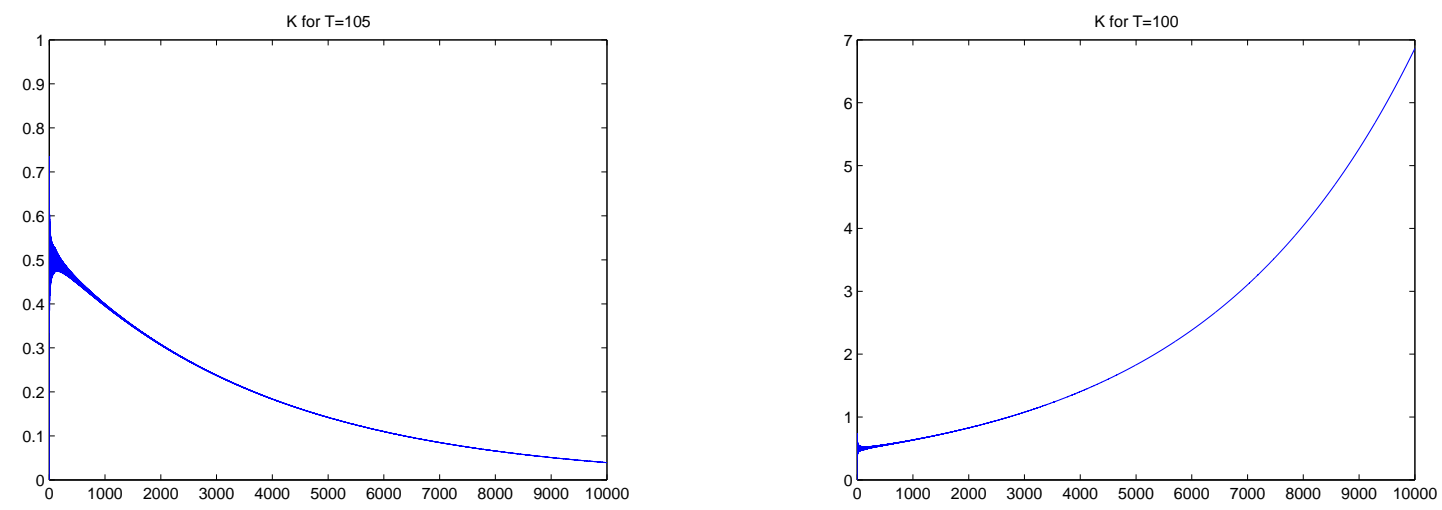

Figure 2: The function $K$ on $[0,10000]$ for $T=105$ and $T=100$

The proof is split into a bunch of lemmas.

The remainder $R$ can be written, for $t \geq T$, as

$$
\begin{aligned}
& R(t)=t^{-k-1 / 2} \cos (2 t-\pi / 4) P_{k}(t)+t^{-k-3 / 2} \sin (2 t-\pi / 4) Q_{k}(t) \\
& P_{k}(t) \sim \sum_{l=0}^{\infty} p_{k l} t^{-2 l}, \quad Q_{k}(t) \sim \sum_{l=0}^{\infty} q_{k l} t^{-2 l}
\end{aligned}
$$

Next we give a representation of $\hat{R}$, which we interpret as the analytic continuation into the left complex half-plane of the Laplace transform of $R$. Recall that $\sigma_{ \pm}= \pm 2$ i.

Lemma 3.5. For $s \in \mathbb{C}$ with $\left|\arg \left(s-\sigma_{ \pm}\right)\right|<\pi$ and $T \rightarrow \infty$, we have, with a function $F$ defined in (A.1),

$$
\begin{aligned}
\hat{R}(s)= & \frac{p_{k 0}}{2} e^{-\mathrm{i} \pi / 4} F\left(-k-1 / 2, s-\sigma_{+}, T\right)+\frac{p_{k 0}}{2} e^{\mathrm{i} \pi / 4} F\left(-k-1 / 2, s-\sigma_{-}, T\right) \\
& +\mathcal{O}\left(e^{-\Re s T} T^{-k-1 / 2}\right), \\
\hat{R}^{\prime}(s)=- & \frac{p_{k 0}}{2} e^{-\mathrm{i} \pi / 4} F\left(-k+1 / 2, s-\sigma_{+}, T\right)-\frac{p_{k 0}}{2} e^{\mathrm{i} \pi / 4} F\left(-k+1 / 2, s-\sigma_{-}, T\right) \\
& +\mathcal{O}\left(e^{-\Re s T} T^{-k+1 / 2}\right) .
\end{aligned}
$$

If $s \in \mathbb{C}$ with $\left|\arg \left(s-\sigma_{ \pm}\right)\right|<\pi$ and $\left|s-\sigma_{ \pm}\right| \geq 1$, then we have the asymptotic expansions

$$
\begin{aligned}
\hat{R}(s)= & \frac{p_{k 0}}{2} e^{-\mathrm{i} \pi / 4}\left(s-\sigma_{+}\right)^{-1} T^{-k-1 / 2} e^{-\left(s-\sigma_{+}\right) T} \\
& \quad+\frac{p_{k 0}}{2} e^{\mathrm{i} \pi / 4}\left(s-\sigma_{-}\right)^{-1} T^{-k-1 / 2} e^{-\left(s-\sigma_{-}\right) T}+\mathcal{O}\left(e^{-\Re s T} T^{-k-3 / 2}\right), \quad T \rightarrow \infty, \\
\hat{R}^{\prime}(s)=- & \frac{p_{k 0}}{2} e^{-\mathrm{i} \pi / 4}\left(s-\sigma_{+}\right)^{-1} T^{-k+1 / 2} e^{-\left(s-\sigma_{+}\right) T} \\
& \quad-\frac{p_{k 0}}{2} e^{\mathrm{i} \pi / 4}\left(s-\sigma_{-}\right)^{-1} T^{-k+1 / 2} e^{-\left(s-\sigma_{-}\right) T}+\mathcal{O}\left(e^{-\Re s T} T^{-k-1 / 2}\right), \quad T \rightarrow \infty .
\end{aligned}
$$


And for $s \in \mathbb{C}$ with $\left|\arg \left(s-\sigma_{+}\right)\right|<\pi$ and $\left|s-\sigma_{+}\right| \leq 1$, we can write

$$
\begin{aligned}
\hat{R}(s) & =\frac{p_{k 0}}{2} e^{-\mathrm{i} \pi / 4} \Gamma(-k+1 / 2) \times \\
& \times\left(\left(s-\sigma_{+}\right)^{k-1 / 2}-\frac{T^{-k+1 / 2}}{\Gamma(-k+1 / 2)} \sum_{n=0}^{\infty} \frac{\left(\left(\sigma_{+}-s\right) T\right)^{n}}{(-k+1 / 2+n) n !}\right)+\mathcal{O}\left(e^{-\Re s T} T^{-k-1 / 2}\right), \\
\hat{R}^{\prime}(s) & =-\frac{p_{k 0}}{2} e^{-\mathrm{i} \pi / 4} \Gamma(-k+3 / 2) \times \\
& \times\left(\left(s-\sigma_{+}\right)^{k-3 / 2}-\frac{T^{-k+3 / 2}}{\Gamma(-k+3 / 2)} \sum_{n=0}^{\infty} \frac{\left(\left(\sigma_{+}-s\right) T\right)^{n}}{(-k+3 / 2+n) n !}\right)+\mathcal{O}\left(e^{-\Re s T} T^{-k+1 / 2}\right) .
\end{aligned}
$$

We have the rough estimates

$$
|\hat{R}(s)| \leq \begin{cases}C_{k} e^{-\Re s T} T^{-k-1 / 2} & : \operatorname{dist}\left(s,\left\{\sigma_{-}, \sigma_{+}\right\}\right) \geq 1 \\ C_{k} e^{-\Re s T} T^{-k+1 / 2} & : \operatorname{dist}\left(s,\left\{\sigma_{-}, \sigma_{+}\right\}\right) \leq 1\end{cases}
$$

and if $s \in \mathbb{C}$ with $\left|\arg \left(s-\sigma_{+}\right)\right|<\pi,\left|s-\sigma_{+}\right| \leq 1$ and $\left|s-\sigma_{+}\right| T \geq M$ for a large constant $M$, then the following refined estimate holds:

$$
|\hat{R}(s)| \leq C_{k, M}\left|s-\sigma_{+}\right|^{-1} T^{-k-1 / 2} e^{-\Re s T} .
$$

Proof. To express the dependence on $k$, we write $R_{k}$ instead of $R$ alone. First, we assume $\Re s \geq 0$. Then formula (3.6) can be obtained from $\hat{R}_{k+2}(s)=\mathcal{O}\left(e^{-\Re s T} T^{-k-3 / 2}\right)$,

$$
\begin{aligned}
\hat{R}_{k}(s)= & p_{k 0} \int_{t=T}^{\infty} e^{-s t} t^{-k-1 / 2} \cos (2 t-\pi / 4) \mathrm{d} t+q_{k 0} \int_{t=T}^{\infty} e^{-s t} t^{-k-3 / 2} \sin (2 t-\pi / 4) \mathrm{d} t \\
& +\hat{R}_{k+2}(s),
\end{aligned}
$$

and (A.10), (A.11). If $s$ is separated from $\sigma_{+}$and $\sigma_{-}$, then the exponent of $T$ in the remainder can be improved by one order. Then (3.8) follows from (3.6) and (A.4); and (3.10) follows from (3.6) and (A.3). The estimates (3.12) and (3.13) are found by application of (3.6), (A.7) and (A.9). The formulas for the derivatives can be obtained observing $F_{\sigma}(\alpha, \sigma, T)=-F(\alpha+1, \sigma, T)$.

Now we consider the case $\Re s<0$. Then the above integrals do not converge, and we have to find a different representation which can be extended to the left half-plane. Therefore, we assume momentarily $\Re s>0$ again. From

$$
\begin{aligned}
\hat{R}_{k}(s)= & \left(\frac{s}{2}-\sqrt{1+\frac{s^{2}}{4}}\right)^{2}-\int_{t=0}^{T} e^{-s t} \mathcal{U}_{11}^{+}(t) \mathrm{d} t \\
& +\frac{1}{\sqrt{\pi}} \sum_{l=0}^{k^{\prime}} \frac{a_{2 l}}{2^{2 l}}\left(e^{-\mathrm{i} \pi / 4} F\left(-3 / 2-2 l, s-\sigma_{+}, T\right)+e^{\mathrm{i} \pi / 4} F\left(-3 / 2-2 l, s-\sigma_{-}, T\right)\right) \\
& -\frac{1}{4 \mathrm{i} \sqrt{\pi}} \sum_{l=0}^{k^{\prime}} \frac{b_{2 l}}{2^{2 l}}\left(e^{-\mathrm{i} \pi / 4} F\left(-5 / 2-2 l, s-\sigma_{+}, T\right)-e^{\mathrm{i} \pi / 4} F\left(-5 / 2-2 l, s-\sigma_{-}, T\right)\right)
\end{aligned}
$$

we directly obtain that $\hat{R}_{k}$ can be analytically extended to the whole complex plane, with the exception of two branch cuts at $\sigma_{-}+\mathbb{R}_{-}$and $\sigma_{+}+\mathbb{R}_{-}$. 
Then the Laplace transform of $R_{k}$ can be expressed, for $\Re s>0$, as

$$
\hat{R}_{k}(s)=-L_{s}(s, T)
$$

where we have defined

$$
L(s, T)=\int_{t=T}^{\infty} M(s, t) \mathrm{d} t, \quad M(s, t)=\frac{e^{-s t}}{t} R_{k}(t) .
$$

A whispering gentle spirit gives us the hint

$$
\partial_{t}\left(\frac{e^{-s t}}{t}\left(J_{2}(2 t)+\frac{2}{s} J_{1}(2 t)\right)\right)=-\frac{e^{-s t}}{s t^{2}}\left(s^{2} t+3 s+4 t\right) J_{2}(2 t)
$$

which can be quickly verified using the relations $J_{\nu}^{\prime}(z)=J_{\nu-1}(z)-\frac{\nu}{z} J_{\nu}(z)$ and $J_{\nu}^{\prime}(z)=$ $-J_{\nu+1}(z)+\frac{\nu}{z} J_{\nu}(z)$. As a consequence, we obtain

$$
\begin{gathered}
\frac{4+s^{2}}{s} M_{s}-3 M=-\frac{e^{-s t}}{s t^{2}}\left(2 s^{2} t+6 s+8 t\right) J_{2}(2 t)-\frac{4+s^{2}}{s} \partial_{s}\left(\frac{e^{-s t}}{t} \tilde{\mathcal{U}}_{11}^{+}(t)\right)+3 \frac{e^{-s t}}{t} \tilde{\mathcal{U}}_{11}^{+}(t) \\
=\partial_{t}\left(e^{-s t}\left(\mathcal{U}_{11}^{+}(t)+\frac{4}{s t} J_{1}(2 t)\right)\right)+\frac{4+s^{2}}{s} e^{-s t} \tilde{\mathcal{U}}_{11}^{+}(t)+3 \frac{e^{-s t}}{t} \tilde{\mathcal{U}}_{11}^{+}(t) .
\end{gathered}
$$

Integrating with respect to $t$ over $[T, \infty)$ then gives

$$
\begin{aligned}
& \frac{4+s^{2}}{s} L_{s}(s, T)-3 L(s, T)=r(s, T) \\
& :=-e^{-s T}\left(\begin{array}{l}
\left.\mathcal{U}_{11}^{+}(T)+\frac{4}{s T} J_{1}(2 T)\right) \\
\quad+\frac{4+s^{2}}{s} \int_{t=T}^{\infty} e^{-s t} \tilde{\mathcal{U}}_{11}^{+}(t) \mathrm{d} t+3 \int_{t=T}^{\infty} \frac{e^{-s t}}{t} \tilde{\mathcal{U}}_{11}^{+}(t) \mathrm{d} t .
\end{array}\right.
\end{aligned}
$$

We solve this ODE, and see that

$$
\begin{aligned}
L(s, T) & =-\left(4+s^{2}\right)^{3 / 2} \int_{\sigma=s}^{\infty} \frac{1}{\left(4+\sigma^{2}\right)^{3 / 2}} \cdot \frac{\sigma r(\sigma, T)}{4+\sigma^{2}} \mathrm{~d} \sigma \\
\hat{R}_{k}(s) & =-L_{s}(s, T)=3 s\left(4+s^{2}\right)^{1 / 2} \int_{\sigma=s}^{\infty} \frac{\sigma r(\sigma, T)}{\left(4+\sigma^{2}\right)^{5 / 2}} \mathrm{~d} \sigma-\frac{s r(s, T)}{4+s^{2}} \\
& =s\left(4+s^{2}\right)^{1 / 2} \int_{\sigma=s}^{\infty} \frac{r_{\sigma}(\sigma, T)}{\left(4+\sigma^{2}\right)^{3 / 2}} \mathrm{~d} \sigma .
\end{aligned}
$$

For a value $\sigma$ in the complex plane with cuts along $\sigma_{-}+\mathbb{R}_{-}$and $\sigma_{+}+\mathbb{R}_{-}$, the terms $\left(4+\sigma^{2}\right)^{\alpha}$ are to be understood as $\left(\sigma-\sigma_{-}\right)^{\alpha}\left(\sigma-\sigma_{+}\right)^{\alpha}$. Then we have an analytic extension of $\hat{R}_{k}$ to the complex plane with the two cuts, via a special choice of the path $\Gamma$ connecting $s$ and $\infty$ : we suppose that $\Gamma$ consists of vertical or horizontal lines, that $\Gamma$ does not intersect the two cuts, that $\Re \sigma \geq \Re s$ for all $\sigma$ on $\Gamma$, and that $\Gamma$ approaches infinity in the sector $\{\arg s<\pi / 4\}$. Additionally, $\Gamma$ can be chosen in such a way that $\operatorname{dist}\left(\Gamma,\left\{\sigma_{-}, \sigma_{+}\right\}\right) \geq 1 / 2$ for $\operatorname{dist}\left(s,\left\{\sigma_{-}, \sigma_{+}\right\}\right) \geq 1 / 2$, and that $\operatorname{dist}\left(\Gamma,\left\{\sigma_{-}, \sigma_{+}\right\}\right) \geq \operatorname{dist}\left(s,\left\{\sigma_{-}, \sigma_{+}\right\}\right)$for $\operatorname{dist}\left(s,\left\{\sigma_{-}, \sigma_{+}\right\}\right) \leq 1 / 2$. Then we try to obtain the estimates (3.12) from either (3.15) or (3.16), where $s \in \mathbb{C}$. 
However, to this end, we need a better description of the function $r$, especially for $s$ in the left half-plane. By partial integration in the definition (3.14) of the function $r$, we have

$$
\begin{gathered}
r(s, T)=-e^{-s T}\left(R_{k}(T)+\frac{4}{s T} J_{1}(2 T)\right)+\frac{4}{s} \int_{t=T}^{\infty} e^{-s t} \tilde{\mathcal{U}}_{11}^{+}(t) \mathrm{d} t \\
+\int_{t=T}^{\infty} e^{-s t} \partial_{t} \tilde{\mathcal{U}}_{11}^{+}(t) \mathrm{d} t+3 \int_{t=T}^{\infty} \frac{e^{-s t}}{t} \tilde{\mathcal{U}}_{11}^{+}(t) \mathrm{d} t .
\end{gathered}
$$

For a partial integration in the first integral on the right, we wish to know the anti-derivative of $\tilde{\mathcal{U}}_{11}^{+}$as well as possible. To this end, we dive into the details of the structure of $\tilde{\mathcal{U}}_{11}^{+}$and $J_{1}$, always assuming $t \geq T$ :

$$
\begin{aligned}
\tilde{\mathcal{U}}_{11}^{+}(t) & =\frac{2}{\sqrt{\pi}} \sum_{l=0}^{(k-3) / 2}\left(\frac{a_{2 l}}{4^{l}} \frac{\cos (2 t-5 \pi / 4)}{t^{2 l+3 / 2}}-\frac{b_{2 l}}{4^{l+1}} \frac{\sin (2 t-5 \pi / 4)}{t^{2 l+5 / 2}}\right), \\
\frac{1}{t} J_{1}(2 t) & \sim \frac{1}{\sqrt{\pi}} \sum_{l=0}^{\infty}\left(\frac{a_{1 l}}{4^{l}} \frac{\cos (2 t-3 \pi / 4)}{t^{2 l+3 / 2}}-\frac{b_{1 l}}{4^{l+1}} \frac{\sin (2 t-3 \pi / 4)}{t^{2 l+5 / 2}}\right),
\end{aligned}
$$

from which we deduce that

$$
\begin{aligned}
\partial_{t}\left(\frac{1}{\sqrt{\pi}}\right. & \left.\sum_{l=0}^{(k-3) / 2}\left(\frac{a_{1 l}}{4^{l}} \frac{\cos (2 t-3 \pi / 4)}{t^{2 l+3 / 2}}-\frac{b_{1 l}}{4^{l+1}} \frac{\sin (2 t-3 \pi / 4)}{t^{2 l+5 / 2}}\right)\right) \\
= & \frac{-2}{\sqrt{\pi}} \sum_{l=0}^{(k-3) / 2}\left(\frac{a_{1 l}}{4^{l}} \frac{\cos (2 t-5 \pi / 4)}{t^{2 l+3 / 2}}-\frac{b_{1 l}}{4^{l+1}} \frac{\sin (2 t-5 \pi / 4)}{t^{2 l+5 / 2}}\right) \\
& -\frac{2}{\sqrt{\pi}} \sum_{l=0}^{(k-3) / 2}\left(-(l+3 / 4) \frac{a_{1 l}}{4^{l}} \frac{\sin (2 t-5 \pi / 4)}{t^{2 l+5 / 2}}-(l+5 / 4) \frac{b_{1 l}}{4^{l+1}} \frac{\cos (2 t-5 \pi / 4)}{t^{2 l+7 / 2}}\right) \\
= & -\tilde{\mathcal{U}}_{11}^{+}(t)+\frac{k-1 / 2}{\sqrt{\pi}} \frac{b_{1,(k-3) / 2}}{2^{k-1}} \frac{\cos (2 t-5 \pi / 4)}{t^{k+1 / 2}}
\end{aligned}
$$

which is found after a tedious calculation convincing us that

$$
\frac{b_{1 l}}{4^{l+1}}+(l+3 / 4) \frac{a_{1 l}}{4^{l}}=\frac{b_{2 l}}{4^{l+1}}, \quad \frac{a_{1, l+1}}{4^{l+1}}-(l+5 / 4) \frac{b_{1 l}}{4^{l+1}}=\frac{a_{2, l+1}}{4^{l+1}} .
$$

Let us write this identity $(3.18)$ as $\partial_{t} N(t)=-\tilde{\mathcal{U}}_{11}^{+}(t)+c t^{-k-1 / 2} \cos (2 t-5 \pi / 4)$. We can then perform the desired partial integration in (3.17), and get

$$
\begin{aligned}
r(s, T)= & -e^{-s T}\left(R_{k}(T)+\frac{4}{s}\left(\frac{1}{T} J_{1}(2 T)-N(T)\right)\right)+\frac{4 c}{s} \int_{t=T}^{\infty} e^{-s t} t^{-k-1 / 2} \cos (2 t-5 \pi / 4) \mathrm{d} t \\
& +\int_{t=T}^{\infty} e^{-s t}\left(-4 N(t)+\partial_{t} \tilde{\mathcal{U}}_{11}^{+}(t)+\frac{3}{t} \tilde{\mathcal{U}}_{11}^{+}(t)\right) \mathrm{d} t .
\end{aligned}
$$

Note that $J_{1}(2 T) / T-N(T)=\mathcal{O}\left(T^{-k-1 / 2}\right)$. By a very similar computation as for (3.18), it can be shown that

$$
-4 N(t)+\partial_{t} \tilde{\mathcal{U}}_{11}^{+}(t)+\frac{3}{t} \tilde{\mathcal{U}}_{11}^{+}(t)=c^{\prime} t^{-k-1 / 2} \sin (2 t-5 \pi / 4) .
$$


Then we can represent $r(s, T)$, also for $s \in \mathbb{C}_{-}$, as

$$
\begin{aligned}
r(s, T)= & -e^{-s T}\left(R_{k}(T)+\frac{4}{s}\left(\frac{1}{T} J_{1}(2 T)-N(T)\right)\right) \\
& -\frac{2 c}{s}\left(e^{-\mathrm{i} \pi / 4} F\left(-k-1 / 2, s-\sigma_{+}, T\right)+e^{\mathrm{i} \pi / 4} F\left(-k-1 / 2, s-\sigma_{-}, T\right)\right) \\
& -\frac{c^{\prime}}{2 \mathrm{i}}\left(e^{-\mathrm{i} \pi / 4} F\left(-k-1 / 2, s-\sigma_{+}, T\right)-e^{\mathrm{i} \pi / 4} F\left(-k-1 / 2, s-\sigma_{-}, T\right)\right) .
\end{aligned}
$$

Plugging this expression into either (3.15) or (3.16), we then quickly obtain (3.12). This completes the proof of Lemma 3.5.

Remark 3.6. For completeness, we mention that

$$
\begin{array}{ll}
\hat{R}(0)=\int_{t=T}^{\infty} \mathcal{U}_{11}^{+}(t) \mathrm{d} t=\frac{1}{T} J_{1}(2 T), & (k=1), \\
\hat{R}(0)=-\frac{p_{k 0}}{2} \sin (2 T-\pi / 4) T^{-k-1 / 2}+\mathcal{O}\left(T^{-k-3 / 2}\right), & (k \geq 1) .
\end{array}
$$

Now we are in a position to study the function $\hat{K}=\hat{K}(s)=1 /\left(s \sqrt{4+s^{2}}+\hat{R}(s)\right)$ and locate its poles. Our goal is to show that $\hat{K}$ has no poles in the right half-plane $\mathbb{C}_{+}$, under conditions on the choice of the cut-off time $T$ and the index $k$, as in Theorem 3.4. It suffices to search for poles in the upper half-plane, by the following observation.

Remark 3.7. We note that $\hat{R}(\bar{s})+f(\bar{s})=\overline{\hat{R}(s)+f(s)}$, which implies that the complex conjugate of a zero of $\hat{R}+f$ is a zero, too.

For technical reasons, we need some bounds on $\hat{R}$ :

Definition 3.8. We define numbers

$$
\begin{aligned}
& \varepsilon_{0}=\|\hat{R}\|_{L^{\infty}(\{s \in \mathbb{C}: \Re s \geq-1 /(5 T)\})}, \quad(k \geq 1), \\
& \varepsilon_{1}=\|\hat{R}\|_{L^{\infty}\left(\left\{s \in \mathbb{C}:\left|s-\sigma_{+}\right| \leq 1, \Re s \geq-1 /(5 T)\right\}\right)}, \quad \quad(k=1), \\
& \varepsilon_{2}=\|\hat{R}\|_{L^{\infty}(\{s \in \mathbb{C}:|s| \leq 1 / T\})}, \quad(k \geq 1) .
\end{aligned}
$$

Further, we define balls $U_{\sigma_{+}, \varepsilon}$ and $U_{0, T}$ in $\mathbb{C}$ as

$$
\begin{aligned}
& U_{\sigma_{+}, \varepsilon}= \begin{cases}\left\{s \in \mathbb{C}:\left|s-\sigma_{+}\right| \leq \min \left(1 / 100, \varepsilon_{1}^{2} / 15\right)\right\} & : k=1, \\
\left\{s \in \mathbb{C}:\left|s-\sigma_{+}\right| \leq 1 / T^{2}\right\} & : k>1,\end{cases} \\
& U_{0, T}=\{s \in \mathbb{C}:|s| \leq 1 / T\}, \quad(k \geq 1) .
\end{aligned}
$$

We remark that (3.6), (A.7) and Lemma A.2 enable us to deduce that

$$
\begin{aligned}
& \varepsilon_{0} \leq C T^{-k+1 / 2}, \\
& \varepsilon_{1} \leq \frac{2 e^{0.2}}{\sqrt{\pi}} T^{-1 / 2}+\mathcal{O}\left(T^{-3 / 2}\right), \\
& \varepsilon_{2} \leq C T^{-k-1 / 2} .
\end{aligned}
$$


Lemma 3.9. If $k \equiv 1 \bmod 4$ and $T$ is large, then $\hat{K}$ has no pole in $U_{\sigma_{+}, \varepsilon}$.

Proof. First, we consider the case $k=1$. Noting that $p_{10}=-2 / \sqrt{\pi}$, we have from (3.10)

$$
\begin{aligned}
\hat{R}(s) & +f(s)=2 e^{-\mathrm{i} \pi / 4}\left(\left(s-\sigma_{+}\right)^{1 / 2}-\frac{T^{-1 / 2}}{\Gamma(-1 / 2)} \sum_{n=0}^{\infty} \frac{\left(\left(\sigma_{+}-s\right) T\right)^{n}}{(-1 / 2+n) n !}\right) \\
& +s \sqrt{s-\sigma_{-}}\left(s-\sigma_{+}\right)^{1 / 2}+\mathcal{O}\left(T^{-3 / 2}\right) .
\end{aligned}
$$

By $\left|s-\sigma_{+}\right|=\mathcal{O}\left(T^{-1}\right)$ due to (3.23), this can be simplified to

$$
\hat{R}(s)+f(s)=-2 e^{-\mathrm{i} \pi / 4} T^{-1 / 2}\left(\left(\left(s-\sigma_{+}\right) T\right)^{1 / 2}-\sum_{n=0}^{\infty} \frac{\left(\left(\sigma_{+}-s\right) T\right)^{n}}{\sqrt{\pi}(2 n-1) n !}\right)+\mathcal{O}\left(T^{-3 / 2}\right) .
$$

We assume $\hat{R}(s)+f(s)=0$ and $\left|s-\sigma_{+}\right| \leq 1 / 100$ as well as $\left|s-\sigma_{+}\right| \leq \varepsilon_{1}^{2} / 15$. Then we show that the modulus of the leading term of the right-hand side of (3.25) has a positive lower bound, which gives a contradiction for large $T$ : put $z=\left(\sigma_{+}-s\right) T$. Then we find, using $|z| \leq 1 / 7$ for large $T$, the remainder estimate

$$
\left|\sum_{n=0}^{\infty} \frac{z^{n}}{\sqrt{\pi}(2 n-1) n !}+\frac{1}{\sqrt{\pi}}\right| \leq \frac{1}{\sqrt{\pi}} \sum_{n=1}^{\infty}|z|^{n} \leq \frac{1}{\sqrt{\pi}} \cdot \frac{1}{6} .
$$

Hence we see that both items in the leading term are in the right half-plane, from which we get

$$
\left|\left(\left(s-\sigma_{+}\right) T\right)^{1 / 2}-\sum_{n=0}^{\infty} \frac{\left(\left(\sigma_{+}-s\right) T\right)^{n}}{\sqrt{\pi}(2 n-1) n !}\right| \geq \frac{1}{\sqrt{\pi}} \cdot \frac{5}{6} .
$$

Then $\hat{R}(s)+f(s)=0$ is impossible in $U_{\sigma_{+}, \varepsilon}$ for $k=1$ and large $T$.

The second case is $k \geq 5$. By (3.10) and $\left|s-\sigma_{+}\right| T=\mathcal{O}\left(T^{-1}\right),\left|s-\sigma_{+}\right|^{k-1 / 2}=\mathcal{O}\left(T^{-2 k+1}\right)$ :

$$
\hat{R}(s)+f(s)=\frac{p_{k 0}}{2} e^{-\mathrm{i} \pi / 4} \frac{T^{-k+1 / 2}}{k-1 / 2}+4 e^{3 \mathrm{i} \pi / 4}\left(s-\sigma_{+}\right)^{1 / 2}+\mathcal{O}\left(T^{-k-1 / 2}\right) .
$$

We look at the arguments of the terms on the right-hand side: since $k \equiv 1 \bmod 4$, the constant $p_{k 0}$ is negative, hence the first term has argument $3 \pi / 4$. The second term has argument between $\pi / 4$ and $5 \pi / 4$, which proves that $\hat{R}(s)+f(s)$ can not be zero in $U_{\sigma_{+}, \varepsilon}$ for large $T$.

Having excluded poles of $\hat{K}$ in a tiny neighbourhood of $\sigma_{+}$, we are now able to study a larger neighbourhood:

Lemma 3.10. The function $\hat{K}=\hat{K}(s)=1 /\left(s \sqrt{4+s^{2}}+\hat{R}(s)\right)$ has no poles in the set $U_{\sigma_{+}, 1,+}=$ $\left\{s \in \mathbb{C}_{+}:\left|s-\sigma_{+}\right| \leq 1, \Re s \geq-1 /(5 T)\right\}$, provided that $k \equiv 1 \bmod 4$, and $T$ is large.

Proof. In the case $k \geq 5$, we assume $\hat{R}(s)+f(s)=0$ for some $s$ in $U_{\sigma_{+}, 1,+}$. By (3.19), we then can conclude that $\varepsilon_{0} \geq|\hat{R}(s)|=|f(s)| \geq \sqrt{3} \sqrt{\left|s-\sigma_{+}\right|}$, from which it follows that $\left|s-\sigma_{+}\right| \leq \varepsilon_{0}^{2} / 3=\mathcal{O}\left(T^{-2 k+1}\right)$, via (3.22). It remains to apply Lemma 3.9. 
And for $k=1$, we show, as before, that (3.20), (3.23), $s \in U_{\sigma_{+}, 1,+}$, and $\hat{R}(s)+f(s)=0$ yield $\left|s-\sigma_{+}\right| \leq \varepsilon_{1}^{2} / 3=4 e^{0.4} /(3 \pi T)+\mathcal{O}\left(T^{-2}\right)$, which gives $\left|s-\sigma_{+}\right| \leq 1 / 100$ for large $T$. Then we have

$$
\varepsilon_{1} \geq|\hat{R}(s)|=|f(s)| \geq 1.99 \cdot \sqrt{3.99} \sqrt{\left|s-\sigma_{+}\right|} \Longrightarrow\left|s-\sigma_{+}\right|<\frac{\varepsilon_{1}^{2}}{15} .
$$

An application of Lemma 3.9 completes the proof.

Next, we search for poles of $\hat{K}$ near zero.

Lemma 3.11. For large $T$ and $k \geq 1$, the function $\hat{K}$ has exactly one pole $s_{0}$ in the set $U_{0, T}$; and $s_{0}$ can be represented as follows:

$$
\left\{\begin{array}{rlrl}
s_{0} & =\frac{p_{k 0} \sin (2 T-\pi / 4)}{4 T^{k+1 / 2}+p_{k 0} T \sin (2 T-\pi / 4)}+\mathcal{O}\left(T^{-k-3 / 2}\right), & & (k=1), \\
s_{0}=\frac{p_{k 0} \sin (2 T-\pi / 4)}{4 T^{k+1 / 2}}+\mathcal{O}\left(T^{-k-3 / 2}\right), & (k>1) .
\end{array}\right.
$$

Proof. Suppose that $\hat{R}(s)+f(s)=0$ and $|s| \leq 1 / T$. Then we have $\varepsilon_{2} \geq|\hat{R}(s)|=|f(s)| \geq|s|$, via (3.21). From (3.24), we deduce that $s=\mathcal{O}\left(T^{-k-1 / 2}\right)$. Therefore, we investigate the function $\hat{R}+f$ in $U_{0, \varepsilon_{2}}:=\left\{s \in \mathbb{C}:|s| \leq \varepsilon_{2}\right\}$.

First, we observe that $\hat{R}+f$ can not have two different zeroes $s_{1}, s_{2}$ in $U_{0, \varepsilon_{2}}$. Because otherwise, we had $\int_{s=s_{1}}^{s_{2}} f^{\prime}(s) \mathrm{d} s=-\int_{s=s_{1}}^{s_{2}} \hat{R}^{\prime}(s) \mathrm{d} s$, which is impossible for large $T$ because of $f^{\prime}(s) \sim 2$ and $\left|\hat{R}^{\prime}(s)\right| \ll 1$, compare (3.9). By a similar reasoning, this zero (if it exists) must be single. We note that then Remark 3.7 tells us that a zero of $\hat{R}+f$ in $U_{0, \varepsilon_{2}}$ must be real.

Second, we define a number

$$
s_{0}=\frac{1}{2 \pi \mathrm{i}} \oint_{|s|=1 / T} \frac{s\left(\hat{R}^{\prime}(s)+f^{\prime}(s)\right)}{\hat{R}(s)+f(s)} \mathrm{d} s .
$$

If $\left|s_{0}\right|<1 / T$, then $s_{0}$ must be a single zero of the denominator. We define a function $\hat{R}_{0}(s)$ as a part of the first term of the expansion (3.8):

$$
\hat{R}_{(0)}(s):=-\frac{p_{k 0}}{2} e^{-\mathrm{i} \pi / 4} \sigma_{+}^{-1} T^{-k-1 / 2} e^{-\left(s-\sigma_{+}\right) T}-\frac{p_{k 0}}{2} e^{\mathrm{i} \pi / 4} \sigma_{-}^{-1} T^{-k-1 / 2} e^{-\left(s-\sigma_{-}\right) T} .
$$

Then we have, for $|s| \leq 1 / T$, the estimate $\left|\hat{R}(s)-\hat{R}_{(0)}(s)\right| \leq C T^{-k-3 / 2}$, which gives us the possibility for an approximation of $s_{0}$ :

$$
s_{0}=\frac{1}{2 \pi \mathrm{i}} \oint_{|s|=1 / T} \frac{s\left(\hat{R}_{(0)}^{\prime}(s)+2\right)}{\hat{R}_{(0)}(s)+2 s} \mathrm{~d} s+\mathcal{O}\left(T^{-k-3 / 2}\right) .
$$

The first item on the right equals the single zero $s_{1}$ of the denominator, which is found as

$$
s_{1}=\frac{p_{k 0} T^{-k-1 / 2} \sin (2 T-\pi / 4)}{4+p_{k 0} T^{-k+1 / 2} \sin (2 T-\pi / 4)}+\mathcal{O}\left(T^{-k-3 / 2}\right) .
$$

This completes the proof of (3.27). 
Lemma 3.12. If $k \geq 1$ and if $T$ is chosen sufficiently large, then there are no poles of $\hat{K}$ in the set $\{s \in \mathbb{C}: 1 / T \leq|s|$, 凡s $\geq-1 /(5 T)\} \cap\left\{s \in \mathbb{C}:\left|s-\sigma_{ \pm}\right| \geq 1 / 2\right\}$.

Proof. In that set, we have $|f(s)| \gg|\hat{R}(s)|$.

We summarize: the function $\hat{K}$ has in the right half-plane $\{s \in \mathbb{C}: \Re s \geq-1 /(5 T)\}$ exactly three singularities: a single pole at $s_{0}$ given by (3.27), and two branch cut singularities at $\arg \left(s-\sigma_{-}\right)=\pi$ and $\arg \left(s-\sigma_{+}\right)=\pi$. Everywhere else in that half-plane, the function $\hat{K}$ is holomorphic, bounded and continuous up to the line $\Re s=-1 /(5 T)$. Moreover, $\hat{K}(s)$ decays at least like $s^{-2}$ for $s \rightarrow \infty, \Re s \geq-1 /(5 T)$. Then $\hat{K}$ is the Laplace transform of a function $K$ which can be computed via

$$
K(t)=\frac{1}{2 \pi \mathrm{i}} \int_{s=1-\mathrm{i} \infty}^{1+\mathrm{i} \infty} e^{t s} \hat{K}(s) \mathrm{d} s=\frac{1}{2 \pi \mathrm{i}} \int_{s=1-\mathrm{i} \infty}^{1+\mathrm{i} \infty} \frac{e^{t s}}{\hat{R}(s)+f(s)} \mathrm{d} s .
$$

Now let $\Gamma$ be the path connecting $-1 /(10 T)-\mathrm{i} \infty$ and $-1 /(10 T)+\mathrm{i} \infty$ along the line $\Re s=$ $-1 /(10 T)$, with the exceptions of two $\supset$ shaped curves $\Gamma_{-}$and $\Gamma_{+}$circling in counter-clockwise direction around $\sigma_{-}$and $\sigma_{+}$. The advantage of this choice of $\Gamma$ is that (in the case $k=1$ ) the line $\{\Re s=-1 /(10 T)\}$ intersects the balls about $\sigma_{ \pm}$with radius $\varepsilon_{1}^{2} / 15$, in which we have a detailed knowledge about the behaviour of $\hat{R}+f$, from (3.25). The point $s_{0}$ is lying to the right of $\Gamma$ anyway.

Then we can write

$$
\begin{aligned}
(K * R)(t) & =\frac{1}{2 \pi \mathrm{i}} \int_{\Gamma} e^{t s} \frac{\hat{R}(s)}{\hat{R}(s)+f(s)} \mathrm{d} s+\operatorname{Res}_{s_{0}}\left(e^{t s} \frac{\hat{R}(s)}{\hat{R}(s)+f(s)}\right) \\
& =\frac{1}{2 \pi \mathrm{i}} \int_{\Gamma} e^{t s} \frac{\hat{R}(s)}{\hat{R}(s)+f(s)} \mathrm{d} s-s_{0} e^{t s_{0}}\left(1+\mathcal{O}\left(T^{-k+1 / 2}\right)\right) .
\end{aligned}
$$

For sake of brevity, we write the integral as $\int_{\Gamma}=I+I_{+}+I_{-}=\int_{\Re s=-1 /(10 T)}+\int_{\Gamma_{+}}+\int_{\Gamma_{-}}$. By partial integration, also the following representation is valid:

$$
(K * R)(t)=-\frac{1}{2 \pi \mathrm{i} t} \int_{\Gamma} e^{t s} \frac{\hat{R}^{\prime}(s) f(s)-\hat{R}(s) f^{\prime}(s)}{(\hat{R}(s)+f(s))^{2}} \mathrm{~d} s-s_{0} e^{t s_{0}}\left(1+\mathcal{O}\left(T^{-k+1 / 2}\right)\right) ;
$$

and we abbreviate $\int_{\Gamma}=I^{\prime}+I_{+}^{\prime}+I_{-}^{\prime}=\int_{\Re s=-1 /(10 T)}+\int_{\Gamma_{+}}+\int_{\Gamma_{-}}$, as before.

For $\Re s=-1 /(10 T)$, we have $|\hat{R}(s)+f(s)| \geq C|f(s)|$ with some positive number $C$, and therefore

$$
\begin{aligned}
|I| & \leq C e^{-t /(10 T)} T^{-k}, \\
\left|I^{\prime}\right| & \leq C e^{-t /(10 T)} T^{-k+1},
\end{aligned}
$$

which follows from splitting the integration at $-1 /(10 T)+\left\{\sigma_{ \pm}, \pm \mathrm{i}\right\}$ and using (3.12), (3.13).

It remains to consider the integrals on the short curves $\Gamma_{-}$and $\Gamma_{+}$. From (3.25) and (3.26) we get the equivalence $|\hat{R}(s)+f(s)| \sim T^{-k+1 / 2}+\left|s-\sigma_{ \pm}\right|^{1 / 2}$ for $s \in \Gamma_{ \pm}$. We split the integration at the points $\sigma_{ \pm}+2 s_{0}$ and $\sigma_{ \pm}+s_{0}^{\prime}$, tacitly assuming $s_{0}<0$ and setting $s_{0}^{\prime}=-T^{-2 k+1}$ for $k>1$, $s_{0}^{\prime}=2 s_{0}$ for $k=1$. The terms $|\hat{R}(s)| \sim T^{-k+1 / 2}$ and $|f(s)| \sim\left|s-\sigma_{ \pm}\right|^{1 / 2}$ from the denominator 
change their roles at $\sigma_{ \pm}+s_{0}^{\prime}$. We have $-1 /(10 T)<2 s_{0} \leq s_{0}^{\prime}<0$, and we find, making use of the Cauchy integral theorem,

$$
\begin{aligned}
\left|I_{ \pm}\right| \leq C & \int_{\sigma=2\left|s_{0}\right|}^{1 /(10 T)} e^{-t \sigma} \frac{T^{-k+1 / 2}}{T^{-k+1 / 2}+\sigma^{1 / 2}} \mathrm{~d} \sigma+C \int_{\sigma=\left|s_{0}^{\prime}\right|}^{2\left|s_{0}\right|} \frac{T^{-k+1 / 2}}{\sigma^{1 / 2}} \mathrm{~d} \sigma \\
& +C \int_{\sigma=0}^{\left|s_{0}^{\prime}\right|} e^{-t \sigma} \frac{\sigma^{1 / 2}}{T^{-k+1 / 2}} \mathrm{~d} \sigma .
\end{aligned}
$$

If $k=1$, then this can be bounded as

$$
\left|I_{ \pm}\right| \leq \frac{C}{t+T} e^{-2 t\left|s_{0}\right|}+C \min \left(T^{-7 / 4}, T^{-1 / 4} t^{-1}\right) .
$$

And if $k>1$, then

$$
\left|I_{ \pm}\right| \leq C e^{-2 t\left|s_{0}\right|} T^{-k+1 / 2} \min \left(T^{-1 / 2}, T^{k / 2+1 / 4} t^{-1}\right)+C T^{-3 k / 2+1 / 4}+C T^{-2 k+1} .
$$

Moreover, in case of $k=1$, we have the additional direct estimate

$$
\begin{aligned}
\left|I_{ \pm}\right| & =C\left|\int_{\Gamma_{ \pm}} e^{t s} \frac{f(s)}{\hat{R}(s)+f(s)} \mathrm{d} s\right| \leq C T^{1 / 2} \int_{\sigma=0}^{1 /(10 T)} \sigma^{1 / 2} e^{-t \sigma} \mathrm{d} \sigma \leq C T^{1 / 2} \int_{\sigma=0}^{\infty} \sigma^{1 / 2} e^{-t \sigma} \mathrm{d} \sigma \\
& \leq C T^{1 / 2} t^{-3 / 2}
\end{aligned}
$$

It remains to consider the integrals $I_{ \pm}^{\prime}$. For $s \in \Gamma_{ \pm},(3.11)$ implies $\left|\hat{R}^{\prime}(s)\right| \leq C\left|s-\sigma_{ \pm}\right|^{k-3 / 2}+$ $C T^{-k+3 / 2}$, from which we deduce that

$$
\left|I_{ \pm}^{\prime}\right| \leq C \int_{\sigma=0}^{1 /(10 T)} \frac{T^{-k+1 / 2} \sigma^{-1 / 2}}{T^{-2 k+1}+\sigma} \mathrm{d} \sigma
$$

by $\sigma T \leq 1 / 10$. We easily check that $\left|I_{ \pm}^{\prime}\right| \leq C$ for $k \geq 1$.

Then our pointwise estimates on $K * R$ are, under the assumption $s_{0}<0$ :

$$
\begin{array}{cc}
|(K * R)(t)| \leq C e^{-t\left|s_{0}\right|} T^{-3 / 2}+C e^{-t /(10 T)} T^{-1}+\frac{C}{t+T} e^{-2 t\left|s_{0}\right|} & \\
+C \min \left(T^{-7 / 4}, T^{-1 / 4} t^{-1}, T^{1 / 2} t^{-3 / 2}\right), & (k=1), \\
|(K * R)(t)| \leq C e^{-t\left|s_{0}\right|} T^{-k-1 / 2}+C e^{-t /(10 T)} T^{-k} & \\
+C e^{-2 t\left|s_{0}\right|} T^{-k+1 / 2} \min \left(T^{-1 / 2}, T^{k / 2+1 / 4} t^{-1}\right)+C T^{-3 k / 2+1 / 4}, & (k>1), \\
|(K * R)(t)| \leq C e^{-t\left|s_{0}\right|} T^{-k-1 / 2}+C t^{-1}, & (k \geq 1) .
\end{array}
$$

From this it is easy to conclude that

$$
\begin{aligned}
& |(K * R)(t)| \leq \frac{C}{t+T^{k}} \\
& 0 \leq t \leq T^{k+1 / 2}, \\
& |(K * R)(t)| \leq C e^{-t\left|s_{0}\right|} T^{-k}+C T^{-7 / 4}, \\
& |(K * R)(t)| \leq C e^{-t\left|s_{0}\right|} T^{-k-1 / 2}+\frac{C}{t+T^{3 / 2}}, \\
& T^{k+1 / 2} \leq t \leq c_{0} T^{k+1 / 2} \ln T, \quad k=1, \\
& |(K * R)(t)| \leq C e^{-t\left|s_{0}\right|} T^{-k-1 / 2}+\frac{C}{t+T^{7 / 4}}, \\
& T^{k+1 / 2} \leq t \leq c_{0} T^{k+1 / 2} \ln T, \quad k=1, \\
& |(K * R)(t)| \leq C e^{-t\left|s_{0}\right|} T^{-k-1 / 2}+\frac{C}{t+T^{3 k / 2-1 / 4}}, \quad T^{k+1 / 2} \leq t<\infty, \quad k>1 .
\end{aligned}
$$


Here the number $c_{0}$ was chosen in such a way as to ensure that $\exp \left(-2 t\left|s_{0}\right|\right) \leq \exp \left(-t\left|s_{0}\right|\right) T^{-1}$ for $t \geq c_{0} T^{k+1 / 2} \ln T$.

Now we have all necessary estimates to prove the main result.

Proof of Theorem 3.4. The above pointwise estimates quickly yield

$$
\begin{aligned}
\|K * R\|_{L^{1}\left(0, T_{0}\right)} & \leq C \ln \left(1+T_{0} T^{-k}\right), \\
\|K * R\|_{L^{2}\left(0, T_{0}\right)} & \leq C T^{-k / 2}, \\
\|K * R\|_{L^{\infty}\left(0, T_{0}\right)} & \leq C T^{-k},
\end{aligned}
$$

for $0<T_{0}<\infty$ and $k \geq 1$.

\subsection{An Efficient Numerical Scheme}

In this section, we propose an efficient numerical scheme for solving (3.2) with an approximate convolution kernel $\tilde{\mathcal{U}}_{11}^{+}$chosen as in (3.5). The main difficulty lies in evaluating the convolution $g_{(v)}(t)=\left(\tilde{\mathcal{U}}_{11}^{+} * v_{b}\right)(t)$ with an effort considerably smaller than $\mathcal{O}(t)$ for large $t$.

We do not restrict the choice of the method how to solve the ODEs (3.2) for $v_{n}$ with $n \leq b$. Instead, we only make some assumptions:

- the ODEs for $v_{n}, n \leq b$, are solved numerically using a method with fixed step-size $h$ and accuracy $\mathcal{O}\left(h^{q}\right)$,

- $\pi / 2$ is an integer multiple of the time step-size $h$.

The second assumption and the almost-periodicity of the convolution kernel for $t \geq T$ will allow us to compute $g_{(v)}(t)$ be recursion arguments. We put $t_{j}=j h$ for $j=0,1, \ldots, J$; and in the following, we will develop a method for an approximate evaluation of $g_{(v)}\left(t_{j}\right)$ with accuracy $\mathcal{O}\left(h^{q+1}\right)$ and effort $\mathcal{O}(T)$, even for $t_{j} \gg T$.

For the discussion of the error, we need one more assumption:

- for time intervals $I$, the norms $\left\|\partial_{t}^{p} v_{b}\right\|_{L^{\infty}(I)}$ are of similar size, where $p=0, \ldots, q+1$.

Assuming that only a finite number of the initial values $u_{n}^{(0)}, u_{n}^{(1)}$ are nonzero, the function $u_{b}(t)$ has a large time asymptotic behaviour $u_{b}(t) \approx A(t) \cos (2 t-\alpha(t))$, due to $(2.5)$, where $A=A(t)$ and $\alpha=\alpha(t)$ are slowly varying, and $\alpha$ approaches a constant. Then the third assumption is fulfilled if $v_{b}$ oscillates in a similar way as $u_{b}$, which is to be expected for $v_{b} \approx u_{b}$.

In case of $0<t_{j} \leq T$, we can integrate the convolution integral directly. Therefore, we suppose that $t=t_{j}>T$. Naturally, we also assume that the number $k^{\prime}$ from (3.5) is at least zero. 
Setting

$$
\begin{aligned}
& I_{\cos , r, \alpha}(t)=\int_{t^{\prime}=0}^{t-T}\left(t-t^{\prime}\right)^{-r} \cos \left(2\left(t-t^{\prime}\right)-\alpha\right) v_{b}\left(t^{\prime}\right) \mathrm{d} t^{\prime}, \\
& I_{\sin , r, \alpha}(t)=\int_{t^{\prime}=0}^{t-T}\left(t-t^{\prime}\right)^{-r} \sin \left(2\left(t-t^{\prime}\right)-\alpha\right) v_{b}\left(t^{\prime}\right) \mathrm{d} t^{\prime},
\end{aligned}
$$

we can write

$$
\begin{aligned}
g_{(v)}(t)= & \int_{t^{\prime}=0}^{T} \frac{2}{t^{\prime}} J_{2}\left(2 t^{\prime}\right) v_{b}\left(t-t^{\prime}\right) \mathrm{d} t^{\prime} \\
& +\frac{2}{\sqrt{\pi}} \sum_{l=0}^{k^{\prime}}\left(\frac{a_{2 l}}{4^{l}} I_{\cos , 2 l+3 / 2,5 \pi / 4}(t)-\frac{b_{2 l}}{4^{l+1}} I_{\sin , 2 l+5 / 2,5 \pi / 4}(t)\right) .
\end{aligned}
$$

The first integral is harmless. Concerning the $I_{\text {cos }}$ and $I_{\text {sin }}$, we remark that

$$
\begin{aligned}
I_{\cos , r, \alpha}(t & \left.+\frac{\pi}{2}\right)=-\int_{t^{\prime}=0}^{t-T}\left(t-t^{\prime}+\frac{\pi}{2}\right)^{-r} \cos \left(2\left(t-t^{\prime}\right)-\alpha\right) v_{b}\left(t^{\prime}\right) \mathrm{d} t^{\prime} \\
& -\int_{t^{\prime}=t-T}^{t-T+\pi / 2}\left(t-t^{\prime}+\frac{\pi}{2}\right)^{-r} \cos \left(2\left(t-t^{\prime}\right)-\alpha\right) v_{b}\left(t^{\prime}\right) \mathrm{d} t^{\prime} \\
=- & \int_{t^{\prime}=0}^{t-T} \sum_{p=0}^{\infty}\left(\begin{array}{c}
-r \\
p
\end{array}\right)\left(\frac{\pi}{2}\right)^{p}\left(t-t^{\prime}\right)^{-r-p} \cos \left(2\left(t-t^{\prime}\right)-\alpha\right) v_{b}\left(t^{\prime}\right) \mathrm{d} t^{\prime}-\int_{t^{\prime}=t-T}^{t-T+\pi / 2} \ldots \mathrm{d} t^{\prime} \\
=- & \sum_{p=0}^{\infty}\left(\begin{array}{c}
-r \\
p
\end{array}\right)\left(\frac{\pi}{2}\right)^{p} I_{\cos , r+p, \alpha}(t) \\
& -\int_{t^{\prime}=t-T}^{t-T+\pi / 2}\left(t-t^{\prime}+\frac{\pi}{2}\right)^{-r} \cos \left(2\left(t-t^{\prime}\right)-\alpha\right) v_{b}\left(t^{\prime}\right) \mathrm{d} t^{\prime},
\end{aligned}
$$

under the reasonable assumption $\pi /(2 T)<1$. Similarly, we have

$$
\begin{aligned}
I_{\sin , r, \alpha}\left(t+\frac{\pi}{2}\right)=-\sum_{p=0}^{\infty} & \left(\begin{array}{c}
-r \\
p
\end{array}\right)\left(\frac{\pi}{2}\right)^{p} I_{\sin , r+p, \alpha}(t) \\
& -\int_{t^{\prime}=t-T}^{t-T+\pi / 2}\left(t-t^{\prime}+\frac{\pi}{2}\right)^{-r} \sin \left(2\left(t-t^{\prime}\right)-\alpha\right) v_{b}\left(t^{\prime}\right) \mathrm{d} t^{\prime} .
\end{aligned}
$$

The effort of computing the integrals $\int_{t^{\prime}=t-T}^{t-T+\pi / 2} \ldots \mathrm{d} t^{\prime}$ is of course negligable. Choosing a method of order $q+1$ for these two integrals, we get an error of the size

$$
C h^{q+1} T^{-r}\left\|v_{b}\right\|_{C^{q+1}} \leq C_{\int} T^{-r} h^{q+1}\left\|v_{b}\right\|_{L^{\infty}}
$$

by the third assumption.

Recalling that $r \in \mathbb{N}+\frac{1}{2}$, we choose numbers $r_{1}, r_{2} \in \mathbb{N}+\frac{1}{2}$ with $r_{2}>r_{1} \geq 2 k^{\prime}+5 / 2$ appropriately. Our idea is to compute and store all the numbers $I_{\cos / \sin , r, \alpha}\left(t_{j}\right)$ for $j=1, \ldots, J$ and $3 / 2 \leq r \leq r_{2}$. Since $\pi /(2 h)$ is an integer, the terms in the sum with $r+p \leq r_{2}$ have already been computed before. For reasons becoming clear in a moment, we assume $r \leq r_{1}$ in the above recursion formulae (3.29), (3.30).

We are done if we can answer two questions: 
- How to deal with those terms $I_{\cos / \sin , r+p, \alpha}(t)$ of the above sum $\sum_{p=0}^{\infty}$, for which $r+p>r_{2}$ ?

- How to evaluate $I_{\cos / \sin , r, \alpha}(t+\pi / 2)$ when $r_{1}<r \leq r_{2}$ ?

In answering the first question, we exploit Taylor's formula, supposing $2 \tau>\pi$ :

$$
\begin{aligned}
\left(1+\frac{\pi}{2 \tau}\right)^{-r} & =\sum_{p=0}^{P}\left(\begin{array}{c}
-r \\
p
\end{array}\right)\left(\frac{\pi}{2 \tau}\right)^{p}+\left(\begin{array}{c}
-r \\
P+1
\end{array}\right)\left(\frac{\pi}{2 \tau}\right)^{P+1}\left(1+\frac{\pi}{2 \tau^{\prime}(\tau)}\right)^{-r-P-1}, \\
\sum_{p=P+1}^{\infty}\left(\begin{array}{c}
-r \\
p
\end{array}\right)\left(\frac{\pi}{2 \tau}\right)^{p} & =\left(\begin{array}{c}
-r \\
P+1
\end{array}\right)\left(\frac{\pi}{2 \tau}\right)^{P+1}\left(1+\frac{\pi}{2 \tau^{\prime}(\tau)}\right)^{-r-P-1}
\end{aligned}
$$

where $\tau^{\prime}(\tau)$ is an unknown number with $\tau<\tau^{\prime}(\tau)<\infty$. Hence we conclude that

$$
\begin{aligned}
& \sum_{p=r_{2}-r+1}^{\infty}\left(\begin{array}{c}
-r \\
p
\end{array}\right)\left(\frac{\pi}{2}\right)^{p} I_{\cos , r+p, \alpha}(t) \\
& =\left(\begin{array}{c}
-r \\
r_{2}-r+1
\end{array}\right)\left(\frac{\pi}{2}\right)^{r_{2}-r+1} \times \\
& \quad \times \int_{t^{\prime}=0}^{t-T}\left(t-t^{\prime}\right)^{-r_{2}-1}\left(1+\frac{\pi}{2 \tau^{\prime}\left(t-t^{\prime}\right)}\right)^{-r_{2}-1} \cos \left(2\left(t-t^{\prime}\right)-\alpha\right) v_{b}\left(t^{\prime}\right) \mathrm{d} t^{\prime} \\
& \sum_{p=r_{2}-r+1}^{\infty}\left(\begin{array}{c}
-r \\
p
\end{array}\right)\left(\frac{\pi}{2}\right)^{p} I_{\cos , r+p, \alpha}(t)|\leq|\left(\begin{array}{c}
-r \\
r_{2}-r+1
\end{array}\right) \mid\left(\frac{\pi}{2}\right)^{r_{2}-r+1}\left\|v_{b}\right\|_{L^{\infty}} \frac{T^{-r_{2}}}{r_{2}} .
\end{aligned}
$$

The same estimate holds for the terms with $I_{\sin , r+p, \alpha}$. We have to choose the parameters $T, r_{1}$, and $r_{2}$ in such a way that

$$
\left|\left(\begin{array}{c}
-r \\
r_{2}-r+1
\end{array}\right)\right|\left(\frac{\pi}{2}\right)^{r_{2}-r+1} \frac{T^{-r_{2}}}{r_{2}} \leq C_{\int} T^{-r} h^{q+1}, \quad \frac{3}{2} \leq r \leq r_{1}
$$

with $C_{\int}$ from (3.31). We observe that neglecting the tail of the sum $\sum_{p=0}^{\infty}$ in (3.29) and (3.30) is not an option for $r$ close to $r_{2}$, which is the reason why we introduced $r_{1}$ and use (3.29), (3.30) only for $r \leq r_{1}$. We come to the second question:

For $r \geq r_{1}+1$, we have

$$
\begin{aligned}
I_{\cos , r, \alpha}\left(t+\frac{\pi}{2}\right) & =\int_{\tau=T}^{t+\pi / 2} \tau^{-r} \cos (2 \tau-\alpha) v_{b}\left(t+\frac{\pi}{2}-\tau\right) \mathrm{d} \tau \\
& =\int_{\tau=T}^{\gamma_{0} T} \ldots \mathrm{d} \tau+\int_{\tau=\gamma_{0} T}^{t-\pi / 2} \ldots \mathrm{d} \tau, \quad T<\gamma_{0} T<t+\frac{\pi}{2} .
\end{aligned}
$$

If $r_{1}$ is chosen large enough, the integrand is decreasing quickly with growing $\tau$, and the second integral becomes negligable in comparison to the first, for suitably large $\gamma_{0}>1$.

Summing up, we have the following algorithm for the evaluation of $g_{(v)}$ :

- fix $h$ with $\pi /(2 h)=: m \in \mathbb{N}$; fix $T$ with $\sin (2 T-\pi / 4) \approx 1, r_{1}, r_{2} \in \mathbb{N}+1 / 2$ with $2 k^{\prime}+5 / 2 \leq r_{1}<r_{2}$ and (3.32); choose $\gamma_{0}>1$, 
- for $0<t_{j}=j h \leq \gamma_{0} T$ and $3 / 2 \leq r \leq r_{2}$, compute $I_{\cos , r, 5 \pi / 4}\left(t_{j}\right), I_{\sin , r, 5 \pi / 4}\left(t_{j}\right)$ by direct integration; evaluate $g_{(v)}\left(t_{j}\right)$ via $(3.28)$, and compute $v_{b}\left(t_{j+1}\right)$,

- for $\gamma_{0} T<t_{j} \leq T_{0}$, repeat the following:

- for $3 / 2 \leq r \leq r_{1}$, set

$$
\begin{aligned}
I_{\cos , r, 5 \pi / 4}\left(t_{j}\right)=- & \sum_{p=0}^{r_{2}-r}\left(\begin{array}{c}
-r \\
p
\end{array}\right)\left(\frac{\pi}{2}\right)^{p} I_{\cos , r+p, 5 \pi / 4}\left(t_{j-m}\right) \\
& +\int_{t^{\prime}=t_{j}-\pi / 2-T}^{t_{j}-T}\left(t_{j}-t^{\prime}\right)^{-r} \cos \left(2\left(t_{j}-t^{\prime}\right)-5 \pi / 4\right) v_{b}\left(t^{\prime}\right) \mathrm{d} t^{\prime},
\end{aligned}
$$

and accordingly for $I_{\sin , r, 5 \pi / 4}\left(t_{j}\right)$,

- for $r_{1}+1 \leq r \leq r_{2}$, set

$$
I_{\mathrm{cos}, r, 5 \pi / 4}\left(t_{j}\right)=\int_{\tau=T}^{\gamma_{0} T} \tau^{-r} \cos (2 \tau-5 \pi / 4) v_{b}\left(t_{j}-\tau\right) \mathrm{d} \tau,
$$

and accordingly for $I_{\sin , r, 5 \pi / 4}\left(t_{j}\right)$,

- compute $g_{(v)}\left(t_{j}\right)$ by $(3.28)$, and compute $v_{b}\left(t_{j+1}\right)$.

Remark 3.13. For instance, for the choice $T=51$ and $r_{1}=41 / 2, r_{2}=81 / 2$, all the terms $\left|\left(\begin{array}{c}-r \\ r_{2}-r+1\end{array}\right)\right|(\pi / 2)^{r_{2}-r+1} T^{r-r_{2}} / r_{2}$ appearing in (3.32) are less than $10^{-20}$. Then $\gamma_{0}=9$ gives $\gamma_{0}^{-r}<10^{-20}$ too, for $r \geq r_{1}+1$.

\section{A The Incomplete Gamma function and related functions}

In the following, all powers are defined by their principal values with cut along $\mathbb{R}_{-}$.

For $T \in \mathbb{R}_{+}, \alpha \in \mathbb{C}$ and $\sigma \in \mathbb{C}_{+}=\{z \in \mathbb{C}: \Re z>0\}$, we define a function

$$
F=F(\alpha, \sigma, T)=\int_{t=T}^{\infty} e^{-\sigma t} t^{\alpha} \mathrm{d} t
$$

This function can be analytically extended with respect to $T$ into $\mathbb{C} \backslash \mathbb{R}$. . Modifying the path of integration into a vertical line, we obtain the quite rough estimate

$$
|F(\alpha, \sigma, T)| \leq C_{\alpha} e^{-\Re(\sigma T)}|T|^{\alpha+1}, \quad \alpha<-1, \quad \sigma \in \mathbb{R}_{+}, \quad T \in \mathbb{C} \backslash \mathbb{R}_{-} .
$$

Lemma A.1. The function $F$ satisfies the identity

$$
\begin{aligned}
& \frac{1}{\Gamma(\alpha+1)} F(\alpha, \sigma, T) \\
& \quad=T^{\alpha+1}\left((\sigma T)^{-\alpha-1}-\frac{1}{\Gamma(\alpha+1)} \sum_{n=0}^{\infty} \frac{(-\sigma T)^{n}}{(\alpha+1+n) n !}\right), \quad(\alpha, \sigma, T) \in \mathbb{R} \times \mathbb{C}_{+} \times \mathbb{R}_{+},
\end{aligned}
$$

which can be used for an analytic continuation of $F$ to $\mathbb{R} \times\left(\mathbb{C} \backslash \mathbb{R}_{-}\right) \times \mathbb{R}_{+}$. Furthermore, for $T \in \mathbb{R}_{+}$, we have the asymptotic expansion

$$
F(\alpha, \sigma, T)=\sigma^{-1} T^{\alpha} e^{-\sigma T}\left(1+\mathcal{O}\left((\sigma T)^{-1}\right)\right), \quad|\sigma T| \rightarrow \infty, \quad|\arg \sigma|<\frac{3 \pi}{2} .
$$


Proof. We recall from [1] the definition of the incomplete Gamma functions:

$$
\begin{aligned}
& \gamma(a, z)=\int_{t=0}^{z} e^{-t} t^{a-1} \mathrm{~d} t, \quad \Re a>0, \\
& \Gamma(a, z)=\Gamma(a)-\gamma(a, z)=\int_{t=z}^{\infty} e^{-t} t^{a-1} \mathrm{~d} t \\
& \gamma^{*}(a, z)=\frac{z^{-a}}{\Gamma(a)} \gamma(a, z),
\end{aligned}
$$

for which we have the following relations:

$$
\begin{aligned}
& \gamma^{*}(a, z)=e^{-z} \sum_{n=0}^{\infty} \frac{z^{n}}{\Gamma(a+n+1)}=\frac{1}{\Gamma(a)} \sum_{n=0}^{\infty} \frac{(-z)^{n}}{(a+n) n !}, \quad(a, z) \in \mathbb{C} \times \mathbb{C}, \\
& \Gamma(a, z)=z^{a-1} e^{-z}\left(1+\mathcal{O}\left(z^{-1}\right)\right), \quad z \rightarrow \infty, \quad|\arg z|<\frac{3 \pi}{2} .
\end{aligned}
$$

For $\alpha, \sigma \in \mathbb{R}_{+}$, we easily check

$$
\frac{1}{\Gamma(\alpha+1)} F(\alpha, \sigma, T)=\frac{\sigma^{-\alpha-1}}{\Gamma(\alpha+1)} \Gamma(\alpha+1, \sigma T)=\sigma^{-\alpha-1}\left(1-(\sigma T)^{\alpha+1} \gamma^{*}(\alpha+1, \sigma T)\right) .
$$

Analytic continuation gives gives (A.3), and (A.6) yields (A.4).

Substitution shows, first for $T \in \mathbb{R}_{+}, \sigma \in \mathbb{R}_{+}$, the identity $F(\alpha, \sigma, T)=T^{\alpha+1} \sigma^{-\alpha-1} F(\alpha, T, \sigma)$; which holds after analytic extension also for $T \in \mathbb{R}_{+}, \sigma \in \mathbb{C} \backslash \mathbb{R}_{-}$. Then (A.2) implies

$$
|F(\alpha, \sigma, T)| \leq C_{\alpha} e^{-\Re(\sigma T)} T^{\alpha+1}, \quad \alpha<-1, \quad \sigma \in \mathbb{C} \backslash \mathbb{R}_{-}, \quad T \in \mathbb{R}_{+} .
$$

We are going to need a precise knowledge of $C_{\alpha}$ in case of $\alpha=-3 / 2$.

Lemma A.2. For $\alpha=-3 / 2$, (A.7) holds with $C_{\alpha}=2$.

Proof. The easy case is $\Re \sigma \geq 0$, since then the factor $t^{\alpha}$ in the integrand of (A.1) is always real. Setting $z=\sigma T$, we see that the general case is settled if we can prove

$$
\left|e^{z} z^{1 / 2} \Gamma(-1 / 2, z)\right| \leq 2, \quad z \in \mathbb{C} .
$$

This holds for $|z| \geq 20$, as can be shown with (A.6) and a precise discussion of the remainder term as in [8]. The estimate (A.8) holds for real $z \in[-20,0]$, too, which follows from either a careful analysis of (A.5) or numerical calculations. It remains to apply the maximum principle of holomorphic functions.

Remark A.3. We list some more simple properties of the function $F$ :

$$
\begin{aligned}
& F(\alpha, \sigma, T)=\sigma^{-1} T^{\alpha} e^{-\sigma T}+\frac{\alpha}{\sigma} F(\alpha-1, \sigma, T), \\
& \int_{t=T}^{\infty} e^{-s t} t^{\alpha} \cos (2 t-\pi / 4) \mathrm{d} t=\frac{1}{2} e^{-\mathrm{i} \pi / 4} F\left(\alpha, s-\sigma_{+}, T\right)+\frac{1}{2} e^{\mathrm{i} \pi / 4} F\left(\alpha, s-\sigma_{-}, T\right), \\
& \int_{t=T}^{\infty} e^{-s t} t^{\alpha} \sin (2 t-\pi / 4) \mathrm{d} t=\frac{1}{2 \mathrm{i}} e^{-\mathrm{i} \pi / 4} F\left(\alpha, s-\sigma_{+}, T\right)-\frac{1}{2 \mathrm{i}} e^{\mathrm{i} \pi / 4} F\left(\alpha, s-\sigma_{-}, T\right) .
\end{aligned}
$$




\section{References}

[1] M. Abramowitz and I. A. Stegun. Pocketbook of mathematical functions. Abridged edition of "Handbook of mathematical functions". Thun: Verlag Harri Deutsch, 1984.

[2] S. Adelman and J. Doll. Generalized Langevin equation appraoch for atom/solid-surface scattering: collinear atom/harmonic chain model. J. Chem. Phys., 61:4242-4245, 1974.

[3] M. Born and K. Huang. Dynamical theory of crystal lattices. Oxford : Clarendon Press, 1954.

[4] W. Cai, M. de Koning, V. Bulatov, and S. Yip. Minimizing boundary reflections in coupleddomain simulations. Phys. Rev. Lett., 85:3213-3216, 2000.

[5] G. Doetsch. Handbuch der Laplace-Transformation. Birkhäuser, 1971.

[6] W. E and Z. Huang. A dynamic atomistic-continuum method for simulation of crystalline materials. J. Comput. Phys., 182:234-261, 2002.

[7] W. Liu, E. Karpov, and H. Park. Nano Mechanics and Materials: Theory, Multiscale Methods and Applications. Wiley, 2005.

[8] F. W. Olver. Asymptotics and special functions. AKP Classics. Wellesley, 1997.

[9] S. Ren. Electronic states in crystals of finite size : quantum confinement of bloch waves. New York: Springer, 2005.

[10] S. Tang, T. Hou, and W. Liu. A mathematical framework of the bridging scale method. International J. Numer. Methods Engrg., 65:1688-1713, 2006.

[11] S. Tang, T. Hou, and W. Liu. A pseudo-spectral multiscale method: interfacial conditions and coarse grid equations. J. Comput. Phys., 213:57-85, 2006.

[12] G. Wagner and W. Liu. Coupling of atomistic and continuum simulations using a bridging scale decomposition. J. Comput. Phys., 190:249-274, 2003. 